\title{
Average Voltage and Neural Network Based Scheme to Predict Transient Stability Status
}

\author{
Emmanuel Asuming Frimpong ${ }^{1 *}$, Philip Yaw Okyere ${ }^{1}$, and Johnson Asumadu \\ ${ }^{1}$ Department of Electrical and Electronic Engineering, \\ Kwame Nkrumah University of Science and Technology, Ghana \\ ${ }^{2}$ Department of Electrical and Computer Engineering, Western Michigan University, United States \\ "Corresponding author, e-mail: eafrimpong.soe@knust.edu.gh
}

\begin{abstract}
This paper presents a technique that predicts the transient stability status of a power system after a disturbance. It uses generator bus voltage as input parameter and a trained single-input multilayer perceptron neural network (MLPNN) as decision tool. When activated, the scheme samples voltages of all generator buses. Two sets of voltage values are extracted from each sampled generator bus voltage. For each set, the minimum voltage value is obtained. An average value is computed from the minimum voltage values extracted from the first sample sets of the various generator buses. The average value is then used to compute the deviations of the minimum voltage values from the second sets of data. The deviations are then summed and used as input to a trained MLPNN which indicates the stability status. The technique was tested using the IEEE 39-bus test system and its accuracy found to be $98.97 \%$.
\end{abstract}

\section{Keywords : Multilayer perceptron, Neural network, Power system transient stability, Stability prediction} and Transient analysis

\section{Introduction}

Modern power systems are highly interconnected with large generating units. Large disturbances such as the tripping of a transmission line or bus endanger the stability of such systems. The absence of appropriate remedial action in the event of a large disturbance may result in out-ofstep (OS) conditions among generators or generator groups. Large swings of power flows, large separation of generator rotor angles and large fluctuations of voltage and current usually characterize OS conditions [1]. Prolonged OS conditions may result in uncontrolled system separation. An example of this is the wide area disturbance in 2006 to the Union for the Coordination of Transmission of Electricity (UCTE) system which caused it to separate in an uncontrollable way, forming three islands [2]. The formation of uncontrolled islands may lead to blackouts, which in turn result in huge losses to consumers [1], [2]. It has therefore become necessary for power systems to deploy control mechanisms to rapidly respond to large disturbances. Control measures employed include out-of-step blocking and tripping, and controlled islanding [3], [4].
The success of the control measures hinges on early detection of conditions that will lead to transient instability. As a result of this, researchers have developed several methods to detect transient instability timeously and even predict instability with the object of allowing adequate time for control actions [5]-[9]. However, there is the need to improve existing schemes or develop ones that are simpler, more reliable, faster in operation and practically feasible.

In this paper, a scheme to predict transient stability or instability is presented. The scheme utilises the bus voltages of system generators as input parameter. The bus voltages are obtained instantaneously after a bus or line is tripped. The bus voltages are extracted at a rate of 32 samples per cycle. Typically, commercial numerical relays sample at this rate [10]. In the schemes operation, the first sets of voltage samples are processed to determine an average voltage value which serves as a reference voltage. This reference voltage is then applied to the second set of bus voltages to obtain voltage deviations from the reference value. The voltage deviations are summed, and the summed value is then applied to a MLPNN which indicates the stability status of the system. The

Received date 2019-08-01, Revised date 2019-08-01, Accepted date 2019-08-26 
method is simple, accurate, speedy, and can also be practically realized.

\section{Generator bus voltage as input parameter}

Figures 1 and 2 show post-fault voltage trajectories for three-phase bus fault on bus 14 of the test system (described in the section on Test System and Simulations) with varying fault durations. These curves were gotten through dynamic simulation using the Power System Simulator for Engineers (PSS ${ }^{\circledR}$ ) software [11]. The curves in Figure 1 represent the voltage trajectories of the ten generator buses for a threephase fault applied at time $t=0.1 \mathrm{~s}$. The fault was cleared 0.1 seconds later by tripping the faulted bus. The system was stable for this fault condition.

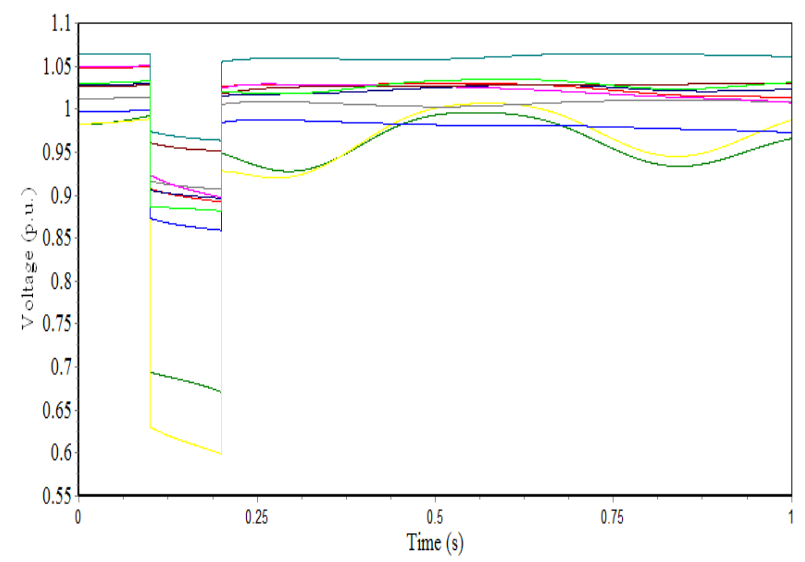

Figure 1. Bus voltage waveforms for a transient stable case

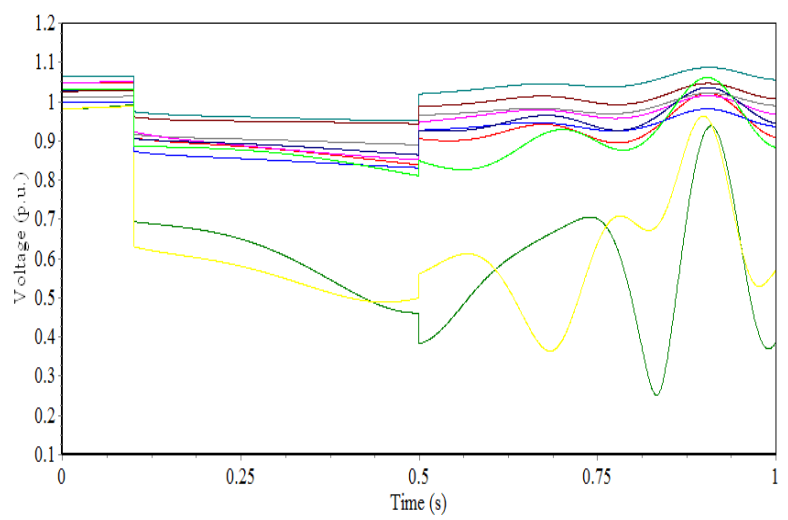

Figure 2. Bus voltage waveforms for a transient unstable case

Conversely, figure 2 shows the post-fault voltage trajectories for the ten generators for the same three-phase bus fault, but which lasted for 0.4 seconds, resulting in transient instability. Comparing the two figures, some significant differences can be observed. For the stable case, all the post-fault voltage trajectories tend to remain somewhat flat. However, for the case resulting in instability, there are significant voltage swings. This difference can be potentially exploited to predict post-fault system stability or instability [9].

The successful use of post-fault bus voltages to predict transient stability or otherwise largely depends on the signal processing approach adopted as well as decision making tool used. This work uses MLPNN for decision making. The approach to signal processing is presented in the section on the proposed transient stability status prediction scheme.

\section{Multilayer perceptron neural network}

MLPNNs are one of the artificial neural networks (ANNs) that have been widely applied in power system studies [3], [4]. They aid in trend identification and pattern realisation that are intricate to be noticed by computer tools or humans [3], [4]. Typically, an MLPNN is systematized interconnected layers of neurons. The layers are: input, hidden and output layers. When a neural cluster in the input layer is provided with data, the neurons propagate the weighted data and any available bias (a bias has a fixed input value of 1), through the hidden layer. The outputs of the hidden layer neurons are then propagated to the output layer where a final response is obtained. Artificial neurons use activation (transfer) functions. The functions usually employed are hyperbolic tangent sigmoid, linear and log-sigmoid [12], [13]. MLPNNs must be trained, just like all other neural networks, [12]. The equation for the output of an MLPNN depends on its architecture. For example, the outputs, $O_{j}$, of a two-layer network with no biases could be expressed as follows:

$$
O_{j}=f\left(\sum_{k} w_{j k}^{(2)} g\left(\sum_{i} w_{i k}^{(1)} x\right)\right)
$$

where $i$ and $\mathrm{j}$ are the neurons in the input and output layer respectively, $g$ and $f$ are the input and output layer transfer functions respectively, and $w^{(1)}$ and $w^{(2)}$ are the weight vectors of the input and output layers respectively. 


\section{Proposed transient stability status prediction scheme}

The proposed scheme predicts transient stability status using the following procedure:

1. Sample voltages of each generator bus at the rate of 32 samples each cycle. For each bus or line voltage, only the initial eight samples are captured and utilized by the proposed algorithm. Using this number of samples results in optimal performance. The sampled voltage for the $n t h$ generator is given as:

$V_{n}=\left\{V_{n}{ }^{1}, V_{n}{ }^{2}, V_{n}{ }^{3}, \ldots, V_{n}{ }^{8}\right\}$

where $n=1,2,3, \ldots, N$ and $N$ is the number of generators.

Each $V_{n}$ is sub-divided into two sample sets $S_{1 n}$ and $S_{2 n}$ with each sample set containing four samples. $S_{1 n}$ comprises the first four samples of $V_{n}$ while $S_{2 n}$ consists of the remaining four samples. $S_{1 n}$ and $S_{2 n}$ are given as follows:

$S_{1 n}=\left\{V_{n}{ }^{1}, V_{n}{ }^{2}, V_{n}{ }^{3}, V_{n}{ }^{4}\right\} n=1,2,3, \ldots, N$

$S_{2 n}=\left\{V_{n}{ }^{5}, V_{n}{ }^{6}, V_{n}{ }^{7}, V_{n}{ }^{8}\right\} n=1,2,3, \ldots, N$

For each $S_{1 n}$ sample set, obtain the minimum value, $X_{n}^{1}$.

$$
X_{n}^{1}=\operatorname{Min}\left(S_{1 n}\right) \quad n=1,2,3, \ldots, N
$$

2. Find the average voltage value, $Y$ of the minimum values in (5) to be used as reference value.

$$
Y=\frac{\sum_{n=1}^{N} X_{n}^{1}}{N} \quad n=1,2,3, \ldots, N
$$

3. Find the minimum value, $X_{n}^{2}$, of each $S_{2 n}$ sample set.

$$
X_{n}^{2}=\operatorname{Min}\left(S_{2 n}\right) \quad n=1,2,3, \ldots, N
$$

4. Obtain the absolute value of the deviation, $D_{n}$, of each minimum value of $S_{2 n}$ from the reference value.

$$
D_{n}=\left|Y-X_{n}^{2}\right| \quad n=1,2,3, \ldots, N
$$

5. Find the sum of the deviations, $S_{D}$.

$$
S_{D}=\sum_{n=1}^{N} D_{n} \quad n=1,2,3, \ldots, N
$$

6. Apply $S_{D}$ as input to the trained MLPNN to obtain predicted stability status, ' $O$ '.

It is expected that $S_{D}$ values for conditions that will lead to transient stability, will be less than $S_{D}$ values for cases that will result in transient instability. The MLPNN should therefore, based on this difference, be able to distinguish between the two cases. Figure 3 portrays the construction of the MLPNN utilized.

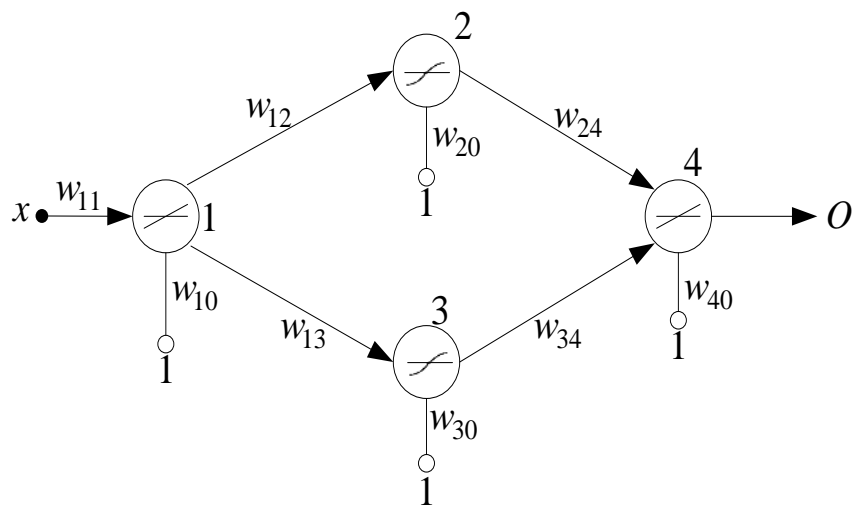

Figure 3. Architecture of used MLPNN

$x=S_{D}$ is the input data, $w_{i j}$ is the weight between neurons $i$ and $j, w_{i 0}$ is the weight of the bias of neuron $i$, and $O$ is the output of the MLPNN. The transfer function of the input and output neurons is linear, and that of the hidden layer neuron is hyperbolic tangent sigmoid.

The output, $y_{1}$, of neuron 1 is given by:

$$
y_{1}=f\left(x w_{11}+w_{10}\right)=x w_{11}+w_{10}
$$

The output, $y_{2}$, of neuron 2 is given by:

$$
\begin{aligned}
y_{2} & =f\left(y_{1} w_{12}+w_{20}\right) \\
& =\frac{e^{2\left(y_{1} w_{12}+w_{20}\right)}-1}{e^{2\left(y_{1} w_{12}+w_{20}\right)}+1} \\
& =\frac{e^{\left.2\left(x w_{11}+w_{10}\right) w_{12}+w_{20}\right)}-1}{e^{2\left(\left(x w_{11}+w_{10}\right) w_{12}+w_{20}\right)}+1}
\end{aligned}
$$

The output, $y_{3}$, of neuron 3 is given by:

$$
\begin{aligned}
y_{3} & =f\left(y_{1} w_{13}+w_{30}\right) \\
& =\frac{e^{2\left(y_{1} w_{13}+w_{30}\right)}-1}{e^{2\left(y_{1} w_{13}+w_{30}\right)}+1} \\
& =\frac{e^{2\left(\left(x w_{11}+w_{10}\right) w_{13}+w_{30}\right)}-1}{e^{2\left(\left(x w_{11}+w_{10}\right) w_{13}+w_{30}\right)}+1}
\end{aligned}
$$

The output, $O$, of MLPNN is thus given by:

Hence,

$$
\begin{aligned}
O & =f\left(y_{2} w_{24}+y_{3} w_{34}+w_{40}\right) \\
& =y_{2} w_{24}+y_{3} w_{34}+w_{40}
\end{aligned}
$$

$$
\begin{aligned}
O= & w_{24} \frac{e^{2\left(\left(x w_{11}+w_{10}\right) w_{12}+w_{20}\right)}-1}{e^{2\left(\left(x w_{11}+w_{10}\right) w_{12}+w_{20}\right)}+1}+ \\
& w_{34} \frac{e^{2\left(\left(x w_{11}+w_{10}\right) w_{13}+w_{30}\right)}-1}{e^{2\left(\left(x w_{11}+w_{10}\right) w_{13}+w_{30}\right)}+1}+w_{40}
\end{aligned}
$$

The MLPNN was trained to produce outputs of either 1 or 0 . A ' 0 ' output predicts stability, while an output of ' 1 ' shows that the system will be unstable. Neural networks seldom output exactly ' 0 ' or ' 1 '. For instance, a desired value ' 0 ' may be obtained as -0.06367 . Consequently, (15) and 
(16) are used in this work to round the MLPNN's output to either 1 or 0 .

$$
\begin{aligned}
& O \geq 0.5 \rightarrow O=1 \\
& O<0.5 \rightarrow O=0
\end{aligned}
$$

\section{Test system and simulations}

Evaluation of the technique was done using the New England study system which is also known as the IEEE 39-bus study system. Figure 4 portrays the system. It is a standard test system extensively utilized for steady state and transient stability studies [4]-[9]. It is made up of 10 generators. One of the generators ( $\mathrm{G} 1$ in figure 4) represents a large system [6]. Data for modelling was taken from [14], [15].

A total of one hundred and ninety-nine threephase line and bus faults were simulated using the $\mathrm{PSS}^{\circledR} \mathrm{E}$ software [11]. The simulations were done by changing the duration of faults and system loading. The system loading simulated were: base load, $20 \%$ reduction, $10 \%$ reduction, $20 \%$ increment and $10 \%$ increment in base load. Faults were made to last for durations of $100 \mathrm{~ms}, 300 \mathrm{~ms}$ and $400 \mathrm{~ms}$. The simulations were done to realize both stable and unstable system states. A criterion espoused in [4] was used to sort the simulations into stable and unstable cases. For a system to be considered as unstable, the rotor angle difference between any two generators should exceed $180^{\circ}, 1$ second after fault clearance [4]. It should be noted that the implementation of the proposed scheme does not require the rotor angles. The sorting yielded 99 and 100 cases of stability and instability respectively. The MLPNN was trained using data from two cases each of stability and instability. Thus, data from 195 cases constituted test data.

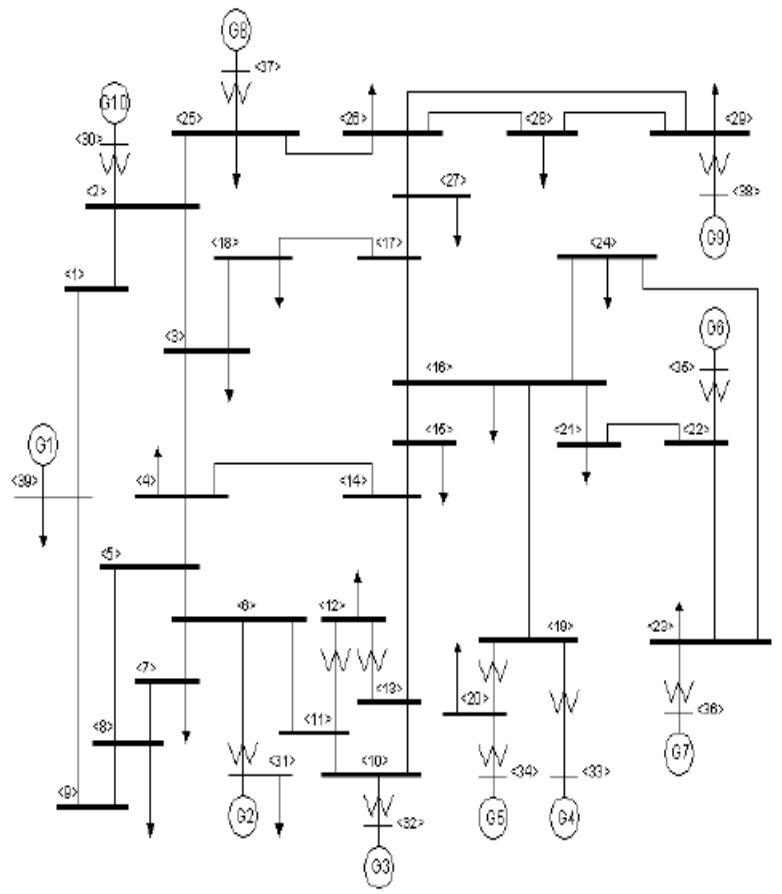

Figure 4. IEEE 39-bus study system

\section{Results}

Overall, the accuracy of the scheme for the 195 test cases was found to be $98.97 \%$. Only two cases were wrongly predicted.

Two representative cases (one each of stable and unstable cases) are presented below to demonstrate the performance of the scheme. Table 1 shows the minimum values of voltages of buses of the 10 generators in the first sample sets $\left(X_{n}^{1}\right)$ and second sample sets $\left(X_{n}^{2}\right)$ for both transient unstable (TU) and transient stable (TS) conditions. The fault condition is a three-phase fault on bus 14 at $110 \%$ base load. The average voltage (reference) values obtained using (6) are as follows:

$$
\begin{gathered}
Y(\text { stable })=0.9996 \\
Y(\text { unstable })=0.84987
\end{gathered}
$$

The corresponding absolute values of deviations from the reference value, $D_{n}$, obtained using (8) are shown in Table 2. Therefore, the sums of deviations obtained using (9) are as follows:

$$
\begin{aligned}
S_{D}(\text { stable }) & =0.2681 \\
S_{D}(\text { unstable }) & =1.1987
\end{aligned}
$$


Table 1. Minimum voltage values for TS and TU cases for first and second sample sets

\begin{tabular}{|c|c|c|c|c|}
\hline \multirow{2}{*}{ Gen. } & \multicolumn{2}{|c|}{ Stable case } & \multicolumn{2}{c|}{ Unstable case } \\
\cline { 2 - 5 } & $X_{n}^{1}(\mathrm{TS})$ & $X_{n}^{2}(\mathrm{TS})$ & $X_{n}^{1}(\mathrm{TU})$ & $X_{n}^{2}(\mathrm{TU})$ \\
\hline G1 & 1.017 & 1.017 & 0.818 & 0.817 \\
\hline G2 & 0.953 & 0.953 & 0.536 & 0.538 \\
\hline G3 & 0.936 & 0.937 & 0.594 & 0.595 \\
\hline G4 & 0.977 & 0.978 & 0.910 & 0.910 \\
\hline G5 & 0.998 & 0.999 & 0.951 & 0.951 \\
\hline G6 & 1.015 & 1.016 & 0.927 & 0.927 \\
\hline G7 & 1.048 & 1.048 & 1.005 & 1.005 \\
\hline G8 & 1.013 & 1.013 & 0.904 & 0.904 \\
\hline G9 & 1.014 & 1.014 & 0.972 & 0.972 \\
\hline G10 & 1.025 & 1.025 & 0.818 & 0.888 \\
\hline
\end{tabular}

Table 2. Absolute values of deviations

\begin{tabular}{|c|c|c|}
\hline Gen. & $D_{n}(\mathrm{TS})$ & $D_{n}(\mathrm{TU})$ \\
\hline G1 & 0.017 & 0.033 \\
\hline G2 & 0.047 & 0.312 \\
\hline G3 & 0.063 & 0.255 \\
\hline G4 & 0.022 & 0.060 \\
\hline G5 & 0.001 & 0.101 \\
\hline G6 & 0.016 & 0.077 \\
\hline G7 & 0.049 & 0.155 \\
\hline G8 & 0.013 & 0.054 \\
\hline G9 & 0.014 & 0.122 \\
\hline G10 & 0.026 & 0.030 \\
\hline
\end{tabular}

Table 3. Weight values of MLPNN.

\begin{tabular}{|c|c|c|}
\hline Input layer & Hidden layer & Output layer \\
\hline$w_{11}=4.1797$ & $w_{12}=-4.6841$ & $w_{24}=-0.54151$ \\
$w_{10}=-1.0008$ & $w_{13}=2.8001$ & $w_{34}=0.054991$ \\
& $w_{20}=3.3579$ & $w_{40}=0.41904$ \\
& $w_{30}=2.8002$ & \\
\hline
\end{tabular}

Table 3 shows the values of interconnecting weights of the MLPNN after training. The outputs of the MLPNN for the two cases are obtained as follows using (10), (11), (12), (13) and (14):

\section{Stable case}

$$
\begin{aligned}
x & =0.2681 \\
y_{1} & =0.119778 \\
y_{2} & =0.992585 \\
y_{3} & =0.996227
\end{aligned}
$$$$
\text { Output } O=-0.06367
$$

Applying (15) the final output is obtained as 0 , which means a stable case.

\section{Unstable case}

$$
\begin{aligned}
x & =1.11987 \\
y_{1} & =4.009406 \\
y_{2} & =-1 \\
y_{3} & =1 \\
\text { Output } O & =1.015541
\end{aligned}
$$

Applying (16), the final output is obtained as 1, which implies an unstable condition.

\section{Conclusion}

A scheme for predicting transient stability status following a disturbance has been presented. It has overall prediction accuracy of $98.97 \%$, which is high. The scheme uses data captured in onefourth of a cycle, which will result in speedy response. Also, the scheme uses input data that can be easily obtained. Another advantage is that the analytical and decision-making tools employed are simple and easy to implement.

\section{References}

[1] K. N. Al-Tallaq and E. A. Feilat, "Online detection of out-of-step operation based on prony analysis-impedance relaying", Proceedings of the 5th WSEAS International Conference on Power Systems and Electromagnetic Compatibility, pp. 55-60, 2005.

[2] P.A. Trodden, W. A. Bukhsh, A. Grothey and K.I.M. McKinnon, "MILP islanding of power networks by bus splitting", Proceedings of IEEE Power and Energy Society General Meeting, 2012.

[3] P. Kundur et al., "Definition and classification of power system stability", IEEE Transactions on Power Systems, vol. 26, no. 2, pp. 1387-1400, 2004.

[4] N. Amjady and S. F. Majedi, "Transient stability prediction by a hybrid intelligent system", IEEE Transaction on Power Systems, vol. 22, no. 3, pp. $1275-1283$, 2007.

[5] E. A. Frimpong, P. Y. Okyere and J. A. Asumadu, "Neural Network and Speed Deviation Based Generator Out-Of-Step Prediction Scheme". Journal of Electrical and Electronic Engineering, vol. 15, no. 2, pp. 1-8, 2015.

[6] D. R. Gurusinghe and A. D. Rajapakse, "Post-Disturbance Transient Stability Status Prediction Using Synchrophasor Measurements", IEEE Transactions on Power Systems, vol. 31, no. 5, pp. 36563664, 2016.

[7] Y. Zhou, J. Wu, Z. Yu, L. Ji and L. Hao. "A Hierarchical Method for Transient Stability 
Prediction of Power Systems Using the Confidence of a SVM-Based Ensemble Classifier", Energies, vol. 9, pp. 1-20, 2016.

[8] A. N. AL-Masri, M. Z. A. A. Kadir, H. Hizam and N. Mariun, "A Novel Implementation for Generator Rotor Angle Stability Prediction Using an Adaptive Artificial Neural Network Application for Dynamic Security Assessment", IEEE Transactions on Power Systems, vol. 28, no. 3, pp. 2516-2525, 2013.

[9] A. D. Rajapakse, F. Gomez, O. M. K. K. Nanayakkara, P. A. Crossley and V. V. Terzija, "Rotor angle stability prediction using post-disturbance voltage trajectory patterns", IEEE Transactions on Power Systems, vol. 25, no. 2, pp. 945-956, 2010.

[10] D. Hou, "Relay Element Performance During Power, System Frequency Excursions", Proceedings of 61st Annual Conference for Protective Relay Engineers, College Station, Texas, 2008.

[11] Power System Simulator for Engineers, PSS $^{\circledR}$ E University Edition, 2016.

[12] A. N. Fathian and M. R. Gholamian, "Using MLP and RBF Neural Networks to Improve the Prediction of Exchange Rate Time Series with ARIMA", International Journal of Information and Electronics Engineering, vol. 2, no. 4, pp. 543-546, 2012.

[13] H. Memarian and S. K. Balasundram, "Comparison between Multi-Layer Perceptron and Radial Basis Function Networks for Sediment Load Estimation in a Tropical Watershed", Journal of Water Resource and Protection, vol. 4, pp. 870876, 2012.

[14] E.A. Frimpong, "Prediction of Transient Stability Status and Coherent Generator Groups". PhD, Department of Electrical and Electronic Engineering, Kwame Nkrumah University of Science and Technology, Kumasi, 2015.

[15] Y. Song, "Design of Secondary Voltage and Stability Controls with Multiple Control Objectives", $\mathrm{PhD}$, School of Electrical and Computer Engineering, Georgia Institute of Technology, Georgia, 2009. 